INPLASY

PROTOCOL

To cite: Xue et al. The efficacy and safety of fire needle for cervical spondylotic radiculopathy: a systematic review and meta-analysis protocol. Inplasy protocol

202170041. doi:

10.37766/inplasy2021.7.0041

Received: 13 July 2021

Published: 13 July 2021

Corresponding author:

Kaiyang Xue

2317649912@qq.com

Author Affiliation:

Guizhou University of

Traditional Chinese Medicine, Guizhou, China.

Support: NO：QZYY-2018-043.

Review Stage at time of this submission: The review has not yet started.

Conflicts of interest:

None declared.

\section{The efficacy and safety of fire needle for cervical spondylotic radiculopathy: a systematic review and meta-analysis protocol}

Xue, K¹ Liu, H²; Shi, Q3; Wang, X4; He, Y5; Cui, J6; Fu, J7.

Review question / Objective: Is fire needle effective and safe in the treatment of cervical spondylotic radiculopathy?

Condition being studied: Cervical spondylotic radiculopathy (CSR).

Information sources: Literature databases: PubMed, EMBASE, Cochrane library, China National Knowledge Infrastructure (CNKI ), Chinese Scientific Journals Database (VIP), Sinomed, Wanfang Database. Trial registry: ClinicalTrials.gov and Chinese Clinic Trials.gov.

INPLASY registration number: This protocol was registered with the International Platform of Registered Systematic Review and Meta-Analysis Protocols (INPLASY) on 13 July 2021 and was last updated on 13 July 2021 (registration number INPLASY202170041).

\section{INTRODUCTION}

Review question / Objective: Is fire needle effective and safe in the treatment of cervical spondylotic radiculopathy?

Condition being studied: Cervical spondylotic radiculopathy (CSR)

\section{METHODS}

Search strategy: The following seven electronic databases and two trial registration platforms will be searched to collect eligible studies published up to June 30, 2021: PubMed, EMBASE, Cochrane library, China National Knowledge Infrastructure (CNKI), Chinese 
Scientific Journals Database (VIP), Sinomed, Wanfang Dat abase, ClinicalTrials.gov and Chinese Clinic Trials.gov. We will use various combination of medical subject Headings (MeSH) and free words to search for terms such as "Cervical spondylotic radiculopathy", " Cervical radiculopathy ", and "fire needle".

Participant or population: Patients with CSR.

Intervention: Two types of RCTs will be included: 1)The experimental group is treated with fire needle alone, while the control group accepts other therapies. 2)The experimental group is treated with fire needle combined with other therapies, while the control group accepts the same other therapies.

Comparator: Two types of RCTs will be included: 1)The experimental group is treated with fire needle alone, while the control group accepts other therapies. 2)The experimental group is treated with fire needle combined with other therapies, while the control group accepts the same other therapies.

Study designs to be included: Randomized controlled trials.

Eligibility criteria: We will include participants who are clearly diagnosed with CSR according to any recognized diagnostic criteria.

Information sources: Literature databases: PubMed, EMBASE, Cochrane library, China National Knowledge Infrastructure (CNKI), Chinese Scientific Journals Database (VIP), Sinomed, Wanfang Database. Trial registry: ClinicalTrials.gov and Chinese Clinic Trials.gov

Main outcome(s): The primary outcome is Visual analogue scale (VAS).

Additional outcome(s): The secondary outcomes are cervical range of motion (ROM), assessment of muscle strength, neck disability index(NDI), the MOS item short from health survey (SF-36), activities of daily living (ADL), total efficiency, and adverse reactions.

Data management: Two researchers will independently complete the data extraction work through a table containing the following information: Publishing features(title, first author, year of publication, Institutional units, journals), Participants and interventions(baseline data, randomization and blinding, treatment site, adverse events, follow-up time), outcomes data. The data will be cross-checked after extraction and any disputes will be resolved by discussion with a third researcher.

Quality assessment / Risk of bias analysis: This study will use the bias risk assessment tool in the Cochrane Handbook 5.1.0 to assess the risk of bias. The content includes 7 items, such as random sequence generation method, allocation concealment method, blinding of clinicians and patients, blinding of outcome evaluators, data integrity, selective reporting, and other sources of bias. According to the literature information, the risk of bias will be judged to be low, high and unclear. Two researchers will assess the risk of bias repeatedly and independently, then cross-check the results. Any differences will be determined by consulting a third party.

Strategy of data synthesis: The Revman5.3 statistical software provided by the Cochrane Collaborative network will be used to summarize and analyze the data included in the literature. Mean difference (MD) will be used as the statistic of continuous data. Relative risk (RR) will be used as the statistic of the dichotomous data. Both continuous data and dichotomous data are expressed in terms of effect size and its $95 \%$ confidence interval (Cl).

Subgroup analysis: Based on the clinical experience in the application of fire needle, when there is significant heterogeneity in the analysis results, we will conduct subgroup analysis according to the basic characteristics of the patients and the 
treatment course to explore the potential sources of heterogeneity.

Sensitivity analysis: In order to verify the robustness of the meta- analysis results, We will remove the included literatures in order of quality from low to high, and recombine the effect sizes to compare with the previous analysis results. If there is no significant change in the results of the combination, the results of the metaanalysis are reliable. If the results of the combination change significantly, the sensitivity is high and the results are not reliable.

Language: No restrictions.

Country(ies) involved: China.

Keywords: fire needle; cervical spondylotic radiculopathy; protocol; systematic review; meta-analysis.

Contributions of each author:

Author 1 - Kaiyang Xue - Writing: original draft; Methodology.

Email: 2317649912@qq.com

Author 2 - Hong Liu - Writing: original draft; Methodology.

Email: 1602412711@qq.com

Author 3 - Qiumei Shi - Investigation.

Email: 2686691604@qq.com

Author 4 - Xianzhu Wang - Investigation.

Email: 2890117835@qq.com

Author 5 - Yanqin He - Investigation.

Email: 1392084489@qq.com

Author 6 - Jin Cui - Writing: review \& editing; Funding acquisition.

Email: 516260179@qq.com

Author 7 - Jing Fu - Conceptualization; Writing: original draft;Writing: review \& editing.

Email: fujing4648@126.com 